\title{
Chronic Phenotype Characterization of a Large- Animal Model of Hereditary Tyrosinemia Type 1
}

Faysal Elgilani, ${ }^{*}$ Shennen A. Mao, ${ }^{\dagger}$ Jaime M. Glorioso, ${ }^{\dagger}$ Meng Yin, ${ }^{\ddagger}$ Ianko D. Iankov, ${ }^{\S}$ Anisha Singh, ${ }^{\dagger}$ Bruce Amiot, Piero Rinaldo, Ronald J. Marler, Richard L. Ehman, Markus Grompe, ${ }^{* *}$ Joseph B. Lillegard, ${ }^{\dagger, \dagger}$ Raymond D. Hickey, ${ }^{\dagger,+\ddagger}$ and Scott L. Nyberg ${ }^{* \dagger}$

From the William J. von Liebig Center for Transplantation and Clinical Regeneration, $*$ the Departments of Surgery, ${ }^{\dagger}$ Radiology, ${ }^{\ddagger}$ and Molecular Medicine, ${ }^{\ddagger}$ and the Division of Laboratory Genetics, ${ }^{\S}$ Department of Laboratory Medicine and Pathology, Mayo Clinic, Rochester, Minnesota; Brami Biomedical, Inc., Minneapolis, Minnesota; the Department of Comparative Medicine," Mayo Clinic, Scottsdale, Arizona; the Papé Family Pediatric Research Institute, ** Oregon Health and Science University, Portland, Oregon; and Midwest Fetal Care Center, ${ }^{\dagger \dagger}$ Children's Hospital and Clinics of Minnesota, Minneapolis, Minnesota

\author{
Accepted for publication \\ September 15, 2016. \\ Address correspondence to \\ Scott L. Nyberg, M.D., Ph.D., \\ William J. von Liebig Center \\ for Transplantation and Clinical \\ Regeneration, Mayo Clinic, 200 \\ First St, Rochester, \\ MN 55905. E-mail: nyberg. \\ scott@mayo.edu.
}

\begin{abstract}
Hereditary tyrosinemia type 1 (HT1) is an autosomal recessive disease caused by deficiency in fumarylacetoacetate hydrolase, the last enzyme in the tyrosine catabolic pathway. In this study, we investigated whether fumarylacetoacetate hydrolase deficient $\left(\mathrm{FAH}^{-1-}\right)$ pigs, a novel large-animal model of HT1, develop fibrosis and cirrhosis characteristic of the human disease. $F A H^{-/-}$pigs were treated with the protective drug 2-(2-nitro-4-trifluoromethylbenzoyl)-1, 3 cyclohexandione (NTBC) at a dose of $1 \mathrm{mg} / \mathrm{kg}$ per day initially after birth. After 30 days, they were assigned to one of three groups based on dosing of NTBC. Group 1 received $\geq 0.2 \mathrm{mg} / \mathrm{kg}$ per day, group 2 cycled on $/$ off NTBC $(0.05 \mathrm{mg} / \mathrm{kg}$ per day $\times 1$ week $/ 0 \mathrm{mg} / \mathrm{kg}$ per day $\times 3$ weeks), and group 3 received no NTBC thereafter. Pigs were monitored for features of liver disease. Animals in group 1 continued to have weight gain and biochemical analyses comparable to wild-type pigs. Animals in group 2 had significant cessation of weight gain, abnormal biochemical test results, and various grades of fibrosis and cirrhosis. No evidence of hepatocellular carcinoma was detected. Group 3 animals declined rapidly, with acute liver failure. In conclusion, the $\mathrm{FAH}^{-1-}$ pig is a large-animal model of HT1 with clinical characteristics that resemble the human phenotype. Under conditions of low-dose NTBC, $F A H^{-1-}$ pigs developed liver fibrosis and portal hypertension, and thus may serve as a large-animal model of chronic liver disease. (Am J Pathol 2017, 187: 33-41; http://dx.doi.org/10.1016/j.ajpath.2016.09.013)
\end{abstract}

Hereditary tyrosinemia type 1 (HT1; Online Mendelian Inheritance in Man number 276700) is an autosomal recessive inborn error of metabolism caused by deficiency of fumarylacetoacetate hydrolase $(F A H)$, the last enzyme in the tyrosine catabolic pathway (Supplemental Figure S1). ${ }^{1}$ Fumarylacetoacetate $(F A A)$, the substrate for $F A H$ in the tyrosine pathway, causes oxidative injury to hepatocytes and proximal renal tubular cells. ${ }^{2-4}$ HT1 has heterogeneous clinical manifestations, including acute liver failure, chronic liver disease, and renal tubular dysfunction. Early treatment with 2-(2-nitro-4trifluoromethylbenzoyl)-1, 3 cyclohexandione (NTBC) reduces the development of liver disease and the need for liver transplantation. ${ }^{5-8}$
Animal models of HT1 are useful research tools to study the mechanisms involved in metabolic liver disorders. $\mathrm{Fah}^{-/-}$ mutant mice, a small-animal model of HT1, have been generated. ${ }^{9,10}$ However, these small-animal models have limited ability to reproduce the similar phenotype as in humans. FAH deficiency is more severe in the mouse than in humans, and hepatocellular carcinoma (HCC) is readily

\footnotetext{
Supported by NIH grant R41 DK092105 (S.L.N.), the Wallace H. Coulter Foundation (S.L.N.), the Marriot Foundation (S.L.N.), the Darwin Deason Family Foundation (S.L.N.), and Mayo Clinic (S.L.N.). The magnetic resonance elastography aspect of the study was supported by NIH grant EB001981 (R.L.E.).

F.E. and S.A.M. have contributed equally to this work.

Disclosures: B.A. is an employee of Brami Biomedical, Inc.
} 
elicited in $\mathrm{Fah}^{-1-}$ mice. ${ }^{11}$ However, $\mathrm{Fah}^{-1-}$ mice do not demonstrate liver fibrosis when subjected to chronic liver injury. ${ }^{12}$ To address the lack of a preclinical model of FAH deficiency, a novel large-animal model was generated by adeno-associated virus-mediated gene knockout and outbreeding of the produced $F A H^{+-}$animals to generate homozygote $F A H^{-1-}$ pigs. ${ }^{13,14}$ Because of their similarity in size, anatomy, and overall metabolic activity to humans, pigs offer improved models for translational biomedical research. ${ }^{15-17}$ As described previously, $F A H^{-1-}$ pigs provide a clinically relevant model of the acute HT1 phenotype. ${ }^{14}$

This study characterizes the chronic phenotype of HT1 in a large-animal model. Given a low maintenance dose of NTBC, $F A H^{-l-}$ pigs demonstrated growth retardation and biochemical abnormalities, such as elevated liver enzymes, tyrosine, succinylacetone (SUAC), and $\alpha$-fetoprotein (AFP). Subsequently, $F A H^{-1-}$ pigs developed chronic liver disease with fibrosis, cirrhosis, and portal hypertension.

\section{Materials and Methods}

\section{Animals and Animal Care}

$F A H^{-1-}$ pigs were produced by mating $F A H^{+/-}$heterozygotes, as described before. ${ }^{13,14}$ Genotyping of all pigs was performed by established PCR and Southern blot assays using ear and tail tissue. ${ }^{14}$ All animals received care according to the criteria outlined by the Institutional Animal Care and Use Committee at Mayo Clinic (Rochester, MN). After delivery, piglets received $1 \mathrm{mg} / \mathrm{kg}$ per day NTBC, in a liquid suspension, for the first 30 days of life (Supplemental Figure S2). $F A H^{-\prime-}$ piglets were initially assigned to one of four dosing regimens $(1.0,0.5,0.2$, and $0 \mathrm{mg} / \mathrm{kg}$ per day). However, based on preliminary observations, $F A H^{-/-}$pigs maintained on 0.2 to $1.0 \mathrm{mg} / \mathrm{kg}$ per day NTBC remained healthy, with normal liver function test results at 3 months of age. Therefore, to reduce the overall cost of NTBC and increase study power, all pigs initially receiving $0.2,0.5$, or $1.0 \mathrm{mg} / \mathrm{kg}$ per day of NTBC were combined in one treatment group (six pigs in group 1). Subsequently, an additional low dose $(0.05 \mathrm{mg} / \mathrm{kg}$ per day with 1 week on, 3 weeks off cycles) NTBC treatment group was added (five pigs in group 2). Pigs that received no NTBC after the first month were classified as group 3 (two pigs). Animals in group 3 had overlap with our former study. ${ }^{13,14}$

\section{Weight Follow-Up}

All animals were weighed daily for the first month, twice weekly up to 3 months of age, and then weekly to the end of the study. Weight gain in $\mathrm{kg}$ per month was determined for each pig, and the average was calculated for all pigs in one group. Comparison of weight gain was made between the different groups. Weight measurements were done over the lifetime of each animal. All animals were observed for clinical symptoms and signs of liver disease and its complications.

\section{Biochemical Analysis}

Pigs were sedated with i.m. injection of $5 \mathrm{mg} / \mathrm{kg}$ telazol and $2 \mathrm{mg} / \mathrm{kg}$ xylazine. Blood was collected from the femoral vein under ultrasound guidance. Serial samples were collected biweekly for the first 3 months and then monthly for the remainder of the study. Standard serum and plasma analyses were performed. Enzyme-linked immunosorbent assay was used to measure AFP. Amino acid was measured in dried blood spots on Whartman 903 Protein Saver Cards (GE Healthcare Ltd., Cardiff, UK) by means of tandem mass spectrometry. ${ }^{18}$

\section{Magnetic Resonance Elastography}

Pigs were sedated with i.m. injection of $5 \mathrm{mg} / \mathrm{kg}$ telazol and $2 \mathrm{mg} / \mathrm{kg}$ xylazine. Intravenous access was obtained in an ear vein, and an endotracheal tube was placed for ventilation and administration of maintenance anesthesia with $2 \%$ to $3 \%$ isoflurane during imaging. Pigs were placed in the supine position and imaged with two acoustic pressureactivated drivers placed against the body wall adjacent to the liver and the spleen. Magnetic resonance elastography (MRE) examinations were performed using a 1.5-Tesla whole-body GE imager (HDx; GE Healthcare, Milwaukee, WI). A four-shot, multislice, spin-echo-based echo planar imaging MRE sequence was used to collect axial wave images. The number of slices ranged from 32 to 48 , depending on animal size. A continuous acoustic vibration at $60 \mathrm{~Hz}$ was transmitted to produce propagated wave images. The acquired wave images were then processed using the direct inversion algorithm ${ }^{19}$ to generate quantitative images of the elastogram. All quantities were reported as means and SDs of regions of interest manually drawn to encompass as much of the liver as possible that had significant wave propagation by visual evaluation. The criteria of region of interest placement were as follows: i) including liver parenchyma only; ii) excluding regions without adequate magnitude signal or shear wave amplitude; iii) keeping a certain distance from the boundaries (at least 3pixel away); and iv) excluding the top and bottom seven slices to obviate edge artifacts. Each MRE imaging session lasted approximately 30 minutes. Pigs were recovered immediately after the scan.

\section{Transabdominal Ultrasound}

Serial abdominal ultrasound scans were obtained every 3 months in all animals under sedation, as described above. Scans were performed by an expert sonographer using a SonoSite-S series machine (SonoSite Inc., Bothell, WA).

\section{Percutaneous Liver Biopsy}

Only pigs in group 2 underwent multiple serial biopsies to correlate the degree of fibrosis to MRE findings. Biopsy 
procedures were conducted during scheduled sedation times for MRE. The pig was placed in the supine position. The right lateral abdomen was prepared with antiseptic, and a $0.5-\mathrm{cm}$ skin incision was made at the midaxillary line. Under ultrasound guidance, a Jamshidi biopsy needle was passed through this incision, through a lower intercostal space, and into the liver parenchyma. After completion of the biopsy, the liver was again evaluated with ultrasound to monitor for complications, specifically hematoma formation. In all groups, liver samples were obtained at the time of euthanasia.

\section{Portal Pressure Measurement}

Before euthanization, three pigs from group 2 underwent an upper midline laparotomy to assess the portal pressure and the hepatic venous pressure gradient. Induction of general anesthesia was achieved with i.m. injection of $5 \mathrm{mg} / \mathrm{kg}$ telazol, $2 \mathrm{mg} / \mathrm{kg}$ xylazine, and $0.01 \mathrm{mg} / \mathrm{kg}$ glycopyrrolate. An i.v. catheter was placed in the ear, and pigs were intubated with an appropriately sized endotracheal tube. Inhaled $1 \%$ to $2 \%$ isoflurane was used for continuous anesthesia during the procedure. After laparotomy and open exposure of the portal and main hepatic veins, an 18-gauge needle was inserted into each vessel. The needle was attached to tubing and a standard blood pressure transducer for measurement of pressures in each vessel. The pigs were euthanized after the procedure.

\section{Histopathologic Analysis}

Histological analysis was performed by a veterinary pathologist (R.J.M.). Tissue samples were fixed in buffered formalin, embedded in paraffin, and stained with hematoxylin and eosin and trichrome stains using standard protocols. FAH immunohistochemistry was performed by using a polyclonal rabbit anti-FAH primary antibody, as previously described. ${ }^{14,20}$ Fibrosis was graded with the modified METAVIR classification scheme, as previously described. $^{21}$

\section{Statistical Analysis}

Baseline variables were described as means \pm SD or medians and interquartile ranges for continuous data. Categorical variables were expressed as counts and percentages. The $t$-test was used to determine statistical significance in normally distributed continuous data. The Wilcoxon test was used for skewed data. Matched-pair continuous data were analyzed with the paired $t$-test and Wilcoxon signed rank test. Continuous variable outcome predictors were determined with simple linear regression and correlated with Pearson's test. JMP version 10 (SAS Institute Inc., Cary, $\mathrm{NC}$ ) was used for analysis.

\section{Results}

\section{Growth Retardation Occurs in $\mathrm{FAH}^{-/-}$Pigs}

As a result of FAH deficiency, liver function and many metabolic processes, including gluconeogenesis, detoxification of ammonia, and synthesis of secreted proteins, are impaired in different animal models. ${ }^{10,14}$ Therefore, we set out to characterize the chronic phenotype of $F A H^{-1-}$ pigs. A total of 13 pigs were included in this study, 6 in group 1, 5 in group 2, and 2 in group 3 (Figure 1 and Supplemental Figure S2). The minimum and maximum durations of follow-up were 3 and 24 months, respectively. We initially monitored weight gain as a parameter of metabolic deficiency in these animals (Figure 2). Animals in groups 2 and 3 ceased to gain weight because of inadequate dosing and withdrawal of NTBC, respectively. In contrast, animals in group 1 continued to gain weight, indicating the adequacy of $\geq 0.2 \mathrm{mg} / \mathrm{kg}$ per day NTBC to prevent development of the HT1 phenotype. Average monthly weight gain was determined for each group and compared to the average weight gain in wild-type (WT) animals in the same institute. Comparison was also made between paired groups. Average weight gain per month was significantly lower in groups 2 and 3 compared to WT pigs, but remained insignificant in group 1 .

\section{$\mathrm{FAH}^{-1-}$ Pigs Have Abnormal Tyrosine and SUAC Levels}

HT1 results from deficiency of FAH, the last enzyme in the catabolic pathway of tyrosine. This causes accumulation of the toxic metabolite FAA. SUAC is a stable derivative of FAA, which can easily be measured. NTBC inhibits 4-hydroxyphenylpyruvic acid dioxygenase and restricts the production of toxic metabolites. Tyrosine accumulates with NTBC treatment as a result of 4-hydroxyphenylpyruvic acid blockage (Supplemental Figure S1). In this study, we used tyrosine and SUAC as surrogate parameters to monitor the effect of NTBC dosing among the three groups. $F A H^{-1-}$ pigs had significantly higher blood tyrosine levels compared to WT pigs (Figure 3A). The variation in tyrosine levels in the treatment groups presumably reflected the differences in NTBC dosage. Although SUAC levels were significantly higher in both groups 2 and 3 than in WT, the difference in levels between group 1 and WT remained insignificant (Figure 3B). Consistent with blood SUAC, the urinary SUAC level was significantly higher in both groups 2 and 3 (Figure 3C). The difference between group 1 and WT was insignificant.

\section{$\mathrm{FAH}^{-/-}$Pigs Have Abnormal Liver Function}

In the human phenotype of HT1, FAA causes oxidative damage to hepatocytes, manifested as abnormal liver function test results. $F A H^{-1-}$ pigs had abnormal biochemical markers, consistent with liver malfunction and injury 


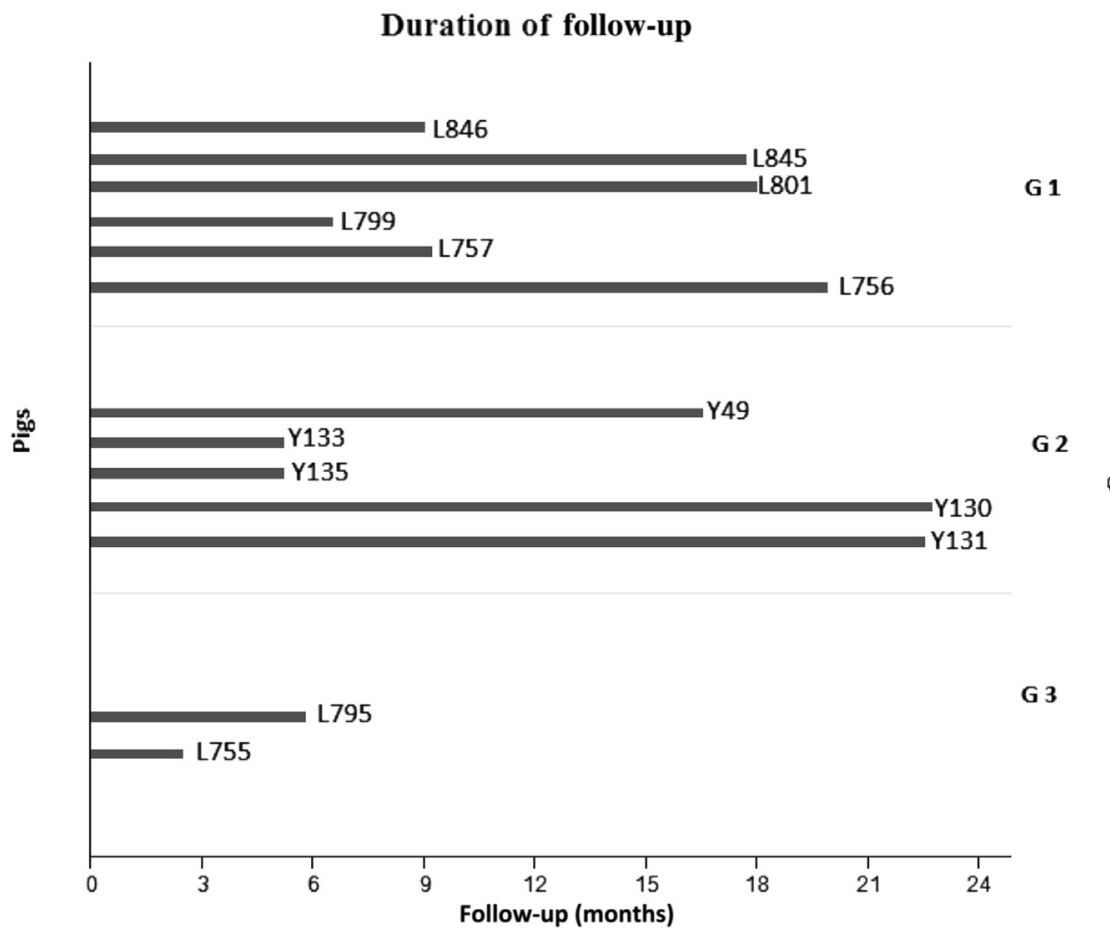

Figure 1 Schematic of timeline for animal experiments. All pigs were maintained on $1.0 \mathrm{mg} / \mathrm{kg}$ per day from birth until 30 days. Pigs were then assigned to three 2-(2-nitro-4-trifluoromethylbenzoyl)-1, 3 cyclohexandione (NTBC) treatment groups. Group (G) 1 received 0.2 to $1 \mathrm{mg} / \mathrm{kg}$ per day NTBC. Group 2 received $0.05 \mathrm{mg} / \mathrm{kg}$ per day with 1 week on and 3 weeks off NTBC cycles. Group 3 had no NTBC administered. $n=6$ pigs (group 1): L846 (male), L845 (female), L801 (female), L799 (female), L757 (male), and L756 (male); $n=5$ pigs (group 2): Y49 (male), Y133 (female), Y135 (male), Y130 (male), and Y131 (female); $n=2$ pigs (group 3): L795 (female) and L755 (male).

(Figure 4 and Table 1). This pattern was more obvious in groups 2 and 3, where inadequate dosage of NTBC was administered. Animals in groups 2 and 3 had multiple biochemical abnormalities consistent with liver injury (Figure 4, A-C). Animals in group 3 demonstrated a more severe pattern, with statistically significant higher levels of aspartate aminotransferase, ammonia, and international

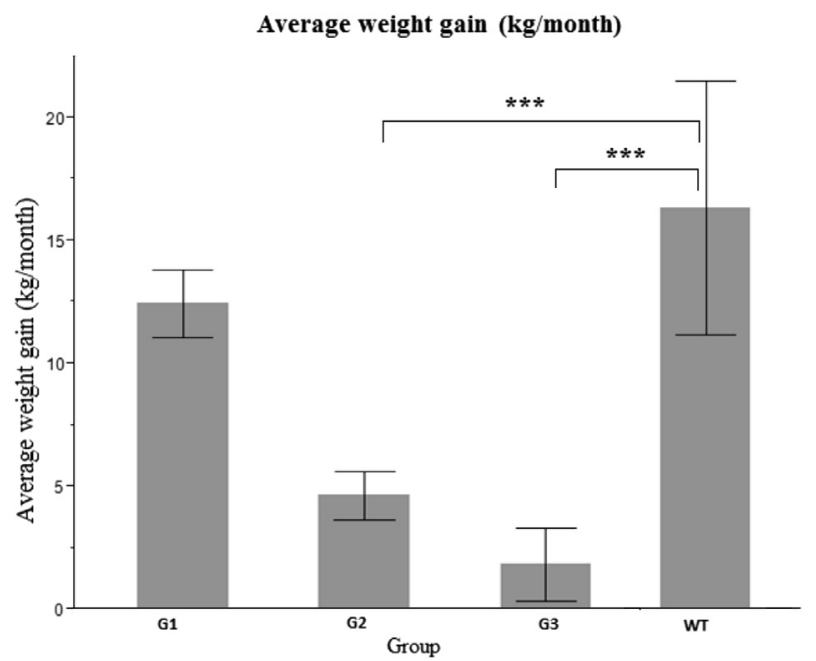

Figure 2 Changes in weight gain in $\mathrm{FAH}^{-/-}$pigs. Weight gain in $\mathrm{kg}$ per month was determined for each pig, and the average was calculated for all pigs in one group. Comparison of average weight gain was made between the different groups. Group (G) 2 and $\mathrm{G} 3$ had significantly less weight gain compared to G1 and wild type (WT). $P$ values represent the significance of difference in weight gain between each group and WT. WT versus G1 was not significant $(P=0.07) .{ }^{* * *} P<0.001$. normalized ratio compared to animals in group 1 and WT. This is consistent with acute liver failure secondary to complete withdrawal of NTBC. Animals in group 2 demonstrated a steady increase in aspartate aminotransferase and ammonia that remained significantly higher than in animals in group 1 and WT. However, the international normalized ratio remained within normal levels among animals in this group. In contrast, animals in group 1, which were adequately treated with NTBC, demonstrated normal values of aspartate aminotransferase, ammonia, and international normalized ratio compared to WT. This is consistent with the protective effect of NTBC.

In addition, we used AFP as a marker for hepatocyte proliferation and development of HCC (Figure 3D and Table 1). Serial blood samples were analyzed every 3 months. AFP was detected in all $F A H^{-1-}$ pigs included in this study (range, 0.2 to $7.7 \mathrm{ng} / \mathrm{mL}$ ). AFP levels remained $<2 \mathrm{ng} / \mathrm{mL}$ during the first 12 months in all pigs. A gradual and significant increase in serum AFP was observed in group 2 pigs after the age of 15 months, coinciding with the onset of advanced liver fibrosis.

\section{$\mathrm{FAH}^{-1-}$ Pigs Undergo Minor Changes in Kidney Function and Histology}

In humans, FAH is strongly expressed in proximal renal tubules, and HT1 is associated with renal impairment. Serial creatinine measurements were used to determine renal function (Figure 4D). $F A H^{-/-}$pigs in both groups 1 and 2 had a higher blood creatinine level than WT pigs; however, 

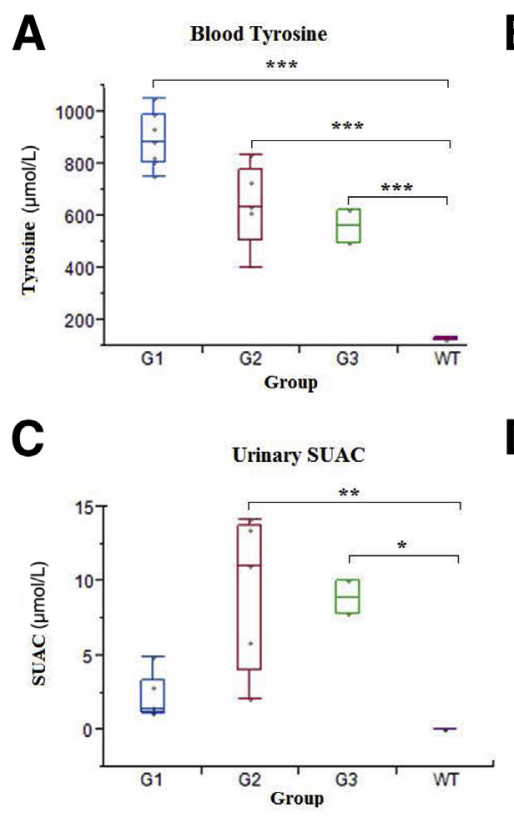

B

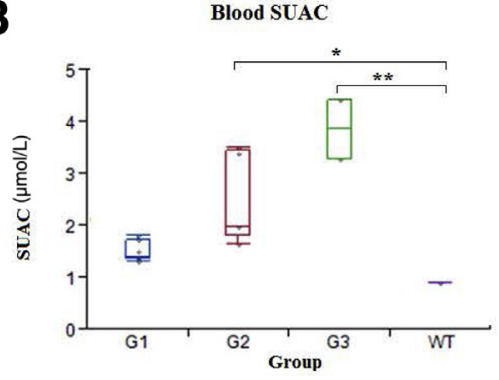

D

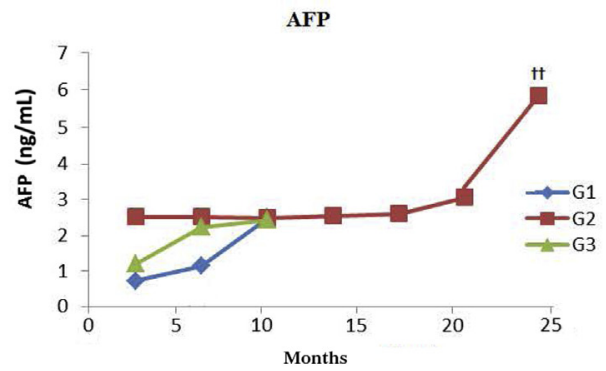

Figure 3 Biochemical abnormalities in $\mathrm{FAH}^{-1-}$ pigs. A: Blood tyrosine levels. Tyrosine levels, as shown in boxplots, were higher in all groups compared to wild type (WT). B: Blood succinylacetone (SUAC) levels. Both group (G) 2 and $\mathrm{G} 3$ had significantly higher levels of SUAC than WT. C: Urinary SUAC levels. Both G2 and G3 had significantly higher levels of urinary SUAC than WT controls. D: $\alpha$-Fetoprotein (AFP) levels. AFP level was significantly higher in G2 when compared to G1. ${ }^{*} P<0.05,{ }^{*} * P<0.01$, and ${ }^{*} * * P 0.001$; ${ }^{\dagger} P<0.01$ between $\mathrm{G} 1$ and $\mathrm{G} 2$. the mean differences were not significant. The lower level of creatinine in group 3 could be attributed to severe loss of muscle mass and liver failure. As group 2 pigs had the longest duration of follow-up, the baseline creatinine and the final creatinine at the time of euthanasia were compared using a matched-pairs $t$-test; however, the difference remained insignificant.

Histological analysis of the kidney was conducted in all animals at the time of euthanasia. Only pigs in group 2 (Supplemental Figure S3) demonstrated abnormalities, which were characterized by tubular dilatation, prominent intraluminal eosinophilic droplets, and occasional sloughed epithelial cells. Tubular epithelial hypertrophy was prominent, with the affected epithelium having abundant eosinophilic cytoplasm. Glomeruli appeared slightly hypercellular, and Bowman's membranes were prominent because of slightly hypertrophied lining epithelium. A mild interstitial fibrosis was scattered throughout.

\section{Progression of Fibrosis Was Monitored Noninvasively in $\mathrm{FAH}^{-/-}$Pigs}

We hypothesized that the low dose of NTBC given to the animals in group 2 would keep them alive but would not
A

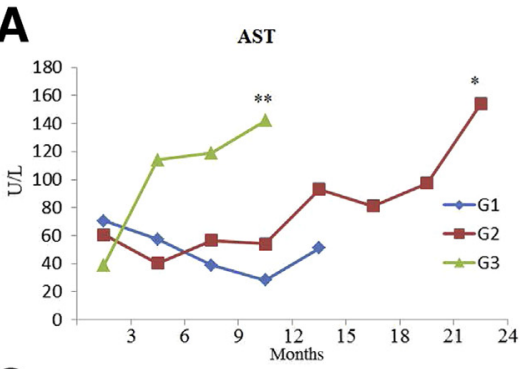

C

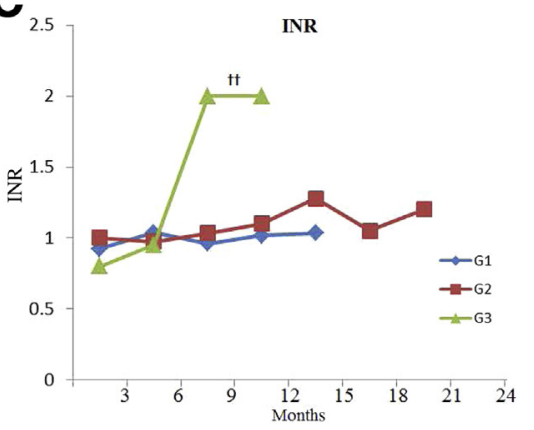

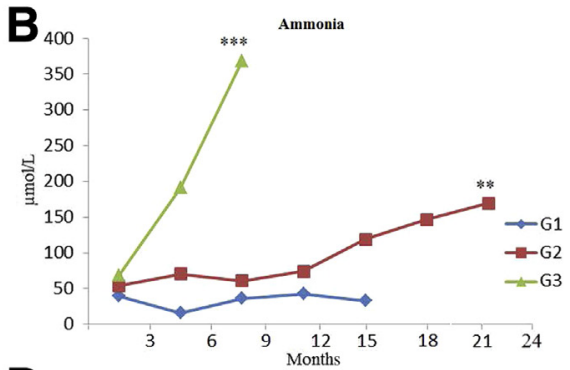

D

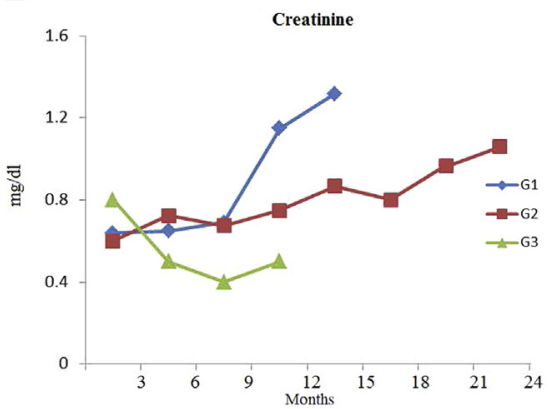

Figure 4 Liver and kidney function in $\mathrm{FAH}^{-/-}$ pigs. A: Aspartate aminotransferase (AST) levels. Groups 2 and 3 demonstrated a more severe pattern of liver injury. AST was significantly higher in groups 2 and 3 than in group 1. B: Ammonia levels. The difference in ammonia level was significant among various groups. Groups 2 and 3 had significantly higher levels compared to group 1. C: International normalized ratio (INR) levels. INR was significantly higher in group 3 compared to groups 1 and 2. No significant difference in INR was detected between groups 1 and 2. D: Creatinine levels. There was no significant difference in creatinine level among the three groups. $\mathrm{G} 1$ versus $\mathrm{G} 2, P=0.9 ; \mathrm{G} 1$ versus $\mathrm{G} 3$, $P=0.1 ; \mathrm{G} 2$ versus $\mathrm{G} 3, P=0.2$. ${ }^{*} P<0.05$, $* * P<0.01$, and $* * * P<0.001$ versus $\mathrm{G} 1$; ${ }^{\dagger \dagger} P<0.01$ versus $\mathrm{G} 1$ and $\mathrm{G} 2$. 
Table 1 Biochemical Analyses

\begin{tabular}{|c|c|c|c|c|}
\hline Biochemical marker & Group 1 & Group 2 & Group 3 & WT \\
\hline SUAC (blood), $\mu \mathrm{mol} / \mathrm{L}$ & $1.5 \pm 0.2$ & $2.5 \pm 0.9^{*}$ & $3.7 \pm 0.4^{* *}$ & $0.9 \pm 0.2$ \\
\hline Tyrosine, $\mu \mathrm{mol} / \mathrm{L}$ & $890 \pm 108^{* * *}$ & $640 \pm 162^{* * *}$ & $596 \pm 90 * * *$ & $132 \pm 3$ \\
\hline SUAC (urine), $\mu \mathrm{mol} / \mathrm{L}$ & $2.1 \pm 1.5$ & $9.3 \pm 5.2^{* *}$ & $8.9 \pm 1.6^{*}$ & $1.1 \pm 01.3$ \\
\hline Ammonia, $\mu \mathrm{mol} / \mathrm{L}$ & $33 \pm 5$ & $84 \pm 18^{* *}$ & $160 \pm 26^{* * *}$ & $32 \pm 7$ \\
\hline AST, U/L & $49 \pm 10$ & $77 \pm 13^{*}$ & $102 \pm 38^{* *}$ & $61 \pm 19$ \\
\hline INR & $1.0 \pm 0.1$ & $1.1 \pm 0.1$ & $1.4 \pm 0.1^{* *}$ & $1.0 \pm 0.1$ \\
\hline Total bilirubin, $\mathrm{mg} / \mathrm{dL}$ & $0.12 \pm 0.07$ & $0.11 \pm 0.04$ & $0.17 \pm 0.10$ & $0.12 \pm 0.04$ \\
\hline Creatinine, $\mathrm{mg} / \mathrm{dL}$ & $0.87 \pm 0.1$ & $0.74 \pm 0.1$ & $0.64 \pm 0.01$ & $0.70 \pm 0.10$ \\
\hline $\mathrm{AFP}, \mathrm{ng} / \mathrm{mL}$ & $0.90 \pm 0.70$ & $2.30 \pm 1.40^{* *}$ & $1.50 \pm 1.00$ & $0.10 \pm 0.06$ \\
\hline
\end{tabular}

Means of biochemical markers in each group were statistically compared to WT pigs as a control. Results expressed as means \pm SD.

$* P<0.05,{ }^{*} P>0.01$, and ${ }^{* * *} P<0.001$.

AFP, $\alpha$-fetoprotein; ALP, alkaline phosphatase; AST, aspartate aminotransferase; INR, international normalized ratio; SUAC, succinylacetone; WT, wild type.

prevent progression of HT1. We used MRE in this study to investigate the influence of portal hypertension and development of fibrosis. This is a noninvasive imaging technology for measuring tissue stiffness by the propagation of shear waves and generating images with quantitative maps of tissue stiffness. Liver and spleen stiffness levels were measured serially, as described above. Four pigs from group 2 underwent monthly MRE to assess progression of liver disease and development of fibrosis (Figure 5). Liver stiffness increased progressively over time and strongly correlated to the duration of the disease. The mean difference between baseline liver stiffness and the final stiffness (before euthanasia at 6 months and 12 months) was statistically significant. Spleen stiffness was measured as an indicator of portal hypertension. The mean difference between baseline and final spleen stiffness was also statistically significant. Stiffness in both liver and spleen showed a strong positive correlation.

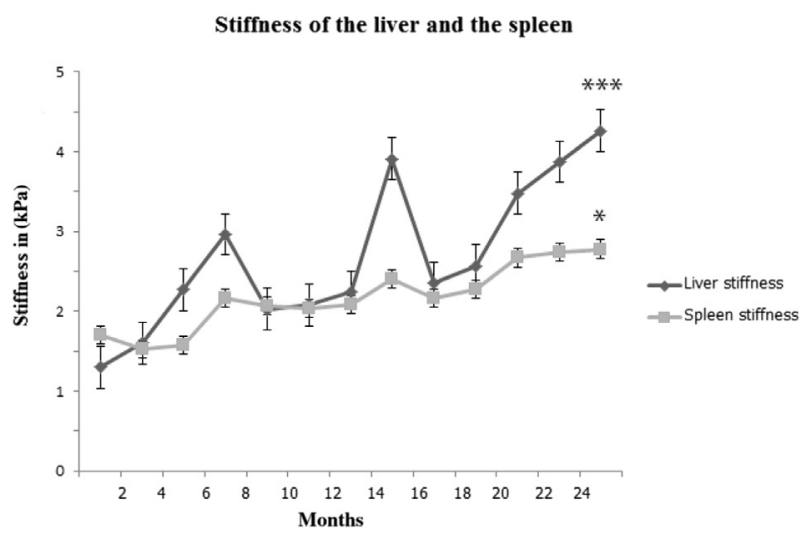

Figure 5 Magnetic resonance elastography (MRE) of the liver and the spleen. MRE was measured in four animals from group 2. Both liver stiffness and spleen stiffness were positively correlated to the duration of the disease. The points on both liver and spleen stiffness lines correspond to the mean stiffness measured at that point of time. The peak in liver stiffness after month 12 corresponds to the time when one of the pigs had a major gastrointestinal bleed. ${ }^{*} P<0.05,{ }^{* * *} P<0.001$ compared to baseline stiffness.
Serial abdominal ultrasound was also performed in all animals under sedation. All animals had a normal liver when scanned after 1 month. Fatty changes were detected at 3 months onward, and most animals developed a coarse pattern and increased liver echogenicity at 6 to 9 months. Splenomegaly and small ascites were detected at 9 months. Liver nodularity and reversal of portal flow were detected on animals that were observed for $>15$ months.

Portal pressure measurements were obtained from three pigs in group 2, one at the age of 18 months and the other two at 24 months (Table 2). All three pigs developed portal hypertension, as indicated by a high hepatic venous pressure gradient of $>5$. The hepatic venous pressure gradient was significantly higher in group 2 compared to three WT pigs. Before euthanasia, all three pigs underwent ultrasound scans, which revealed reversal of portal blood flow, ascites, and splenomegaly.

\section{$\mathrm{FAH}^{-/-}$Pigs Show Histologic Evidence of Liver Fibrosis}

Liver samples were obtained at necropsy from all animals in the three groups (Figure 6). After a follow-up of 18 months, group 1 animals showed no significant pathology

Table 2 Portal Pressure Measurements

\begin{tabular}{|c|c|c|c|c|c|}
\hline \multirow{2}{*}{$\begin{array}{l}\text { Pressure } \\
\text { measurement }\end{array}$} & \multicolumn{3}{|c|}{ Group 2} & \multirow[b]{2}{*}{ Means \pm SD } & \multirow[b]{2}{*}{ WT $(n=3)$} \\
\hline & Pig 1 & Pig 2 & Pig 3 & & \\
\hline PVP & 18 & 18 & 14 & $16.7 \pm 2.3^{* * *}$ & $5.0 \pm 1.0$ \\
\hline HVP & 8 & 10 & 8 & $8.0 \pm 2.0^{*}$ & $3.3 \pm 1.2$ \\
\hline HVPG & 10 & 8 & 8 & $8.7 \pm 1.2^{* * *}$ & $1.7 \pm 0.6$ \\
\hline
\end{tabular}

Direct portal pressure measurements (in $\mathrm{mmHg}$ ) were collected from three pigs in group 2 and three WT pigs. HVPG and PVP were significantly higher in group 2 animals compared to WT. Results expressed as means \pm SD.

${ }^{*} P<0.05,{ }^{* * *} P<0.001$.

HVP, hepatic vein pressure; HVPG, hepatic venous pressure gradient; PVP, portal vein pressure; WT, wild type. 

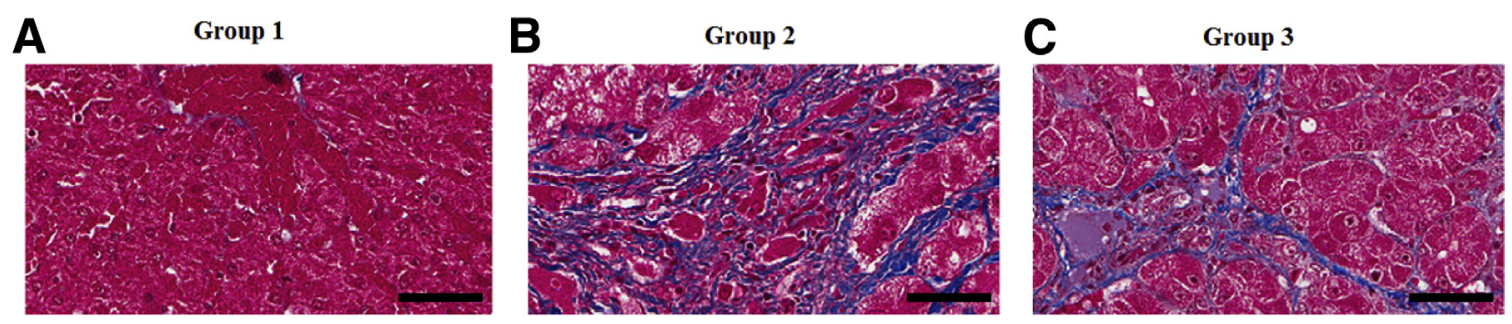

D

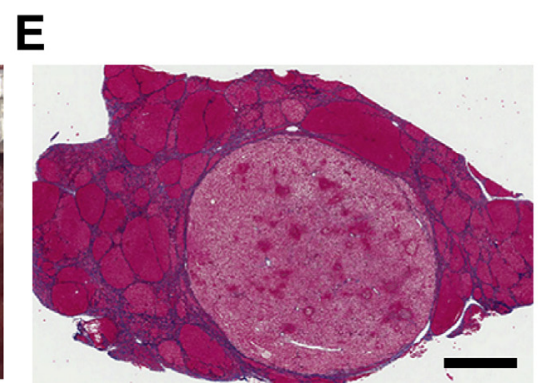

Figure 6 Liver fibrosis and nodularity in $\mathrm{FAH}^{-/-}$pigs. A: Representative trichrome stain shows minimal fibrosis in group 1. B: Representative trichrome stain in group 2 shows extensive interlobular fibrosis with distortion of normal lobular architecture, prominent hepatocellular hypertrophy, and abnormal lobular formation, which appears as multiple nodules. C: Representative trichrome stain in group 3. Histology shows increased interlobular fibrosis with expansion into the hepatic parenchyma. D: Macroscopic appearance of $\mathrm{FAH}^{-/-}$pig from group 2 shows liver hypertrophy and nodularity (scale in centimeters). E: Trichrome stain from group 2 pig shows abnormal lobular formation appearing as multiple nodules. Prominent bands of fibrosis surround clusters of irregularly sized hepatic lobules, consistent with advanced fibrosis. Scale bars: $200 \mu \mathrm{m}(\mathbf{A}-\mathbf{C}) ; 4 \mathrm{~mm}(\mathbf{E})$.

(Figure 6A); four animals had a modified METAVIR fibrosis score of 0 to 1 , and only one animal had a fibrosis score of 3 . Histologic findings in group 3 animals were consistent with increased interlobular fibrosis with some expansion into the hepatic parenchyma (Figure 6C). In group 2 animals, histology showed considerable distortion of normal lobular architecture primarily because of extensive interlobular fibrosis (Figure 6B). Prominent bands of fibrosis surrounded clusters

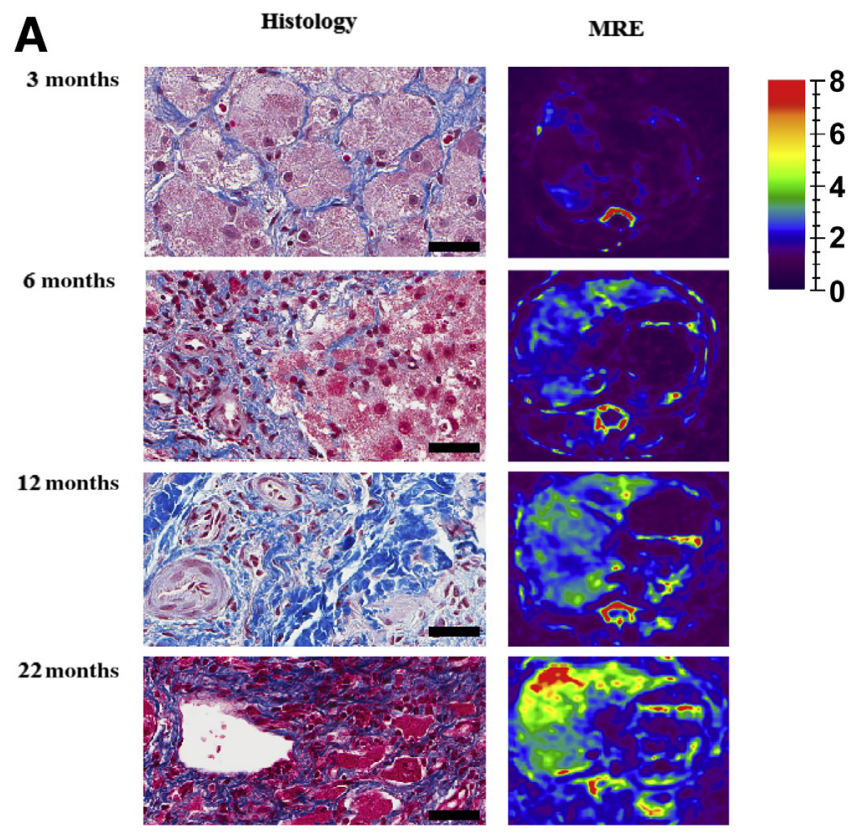

\section{B}

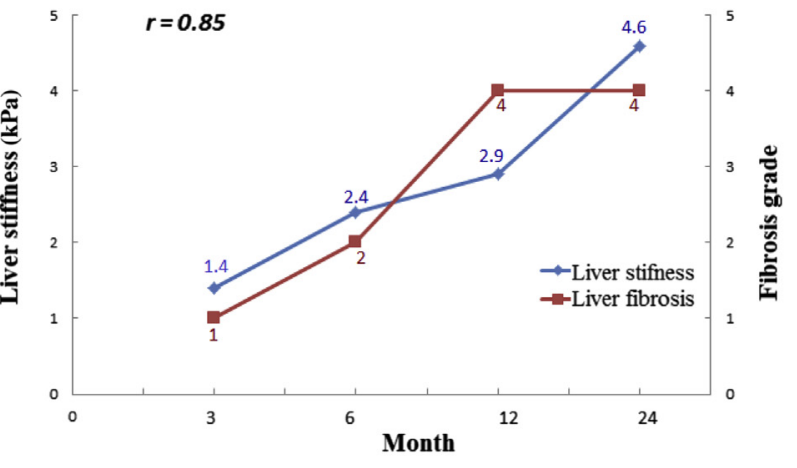

Figure 7 Liver fibrosis and stiffness measurements in one pig from group 2. A: Representative trichrome stains show liver fibrosis in serial biopsies performed at 3, 6, 12, and 22 months, with corresponding magnetic resonance elastography (MRE) images of liver stiffness measurements. The color map used for the stiffness images is shown in the column on the right. B: Correlation plot of change in liver stiffness in $\mathrm{kPa}$ and liver fibrosis grade $(r=0.85)$. Fibrosis grade based on modified METAVIR score: 0 , no increase in fibrosis; 1 , fine bands of fibrosis extending into the surrounding hepatic parenchyma; 2 , bands of fibrosis extending into the surrounding hepatic parenchyma and isolating hepatocytes; 3 , expansion of existing fibrous bands with prominent extension into the hepatic parenchyma; 4, extensive expansion of existing fibrous bands, resulting in clusters of irregularly sized hepatic lobules, often with some periportalcentral venous bridging fibrosis. Scale bars $=200 \mu \mathrm{m}(\mathbf{A})$. 
of irregularly sized hepatic lobules. Hepatocellular hypertrophy was prominent in affected lobules. Fibrosis had progressed with time to grade 3 at 18 months and to grade 4 at 22 months. Extensive nodularity was noted in this group (Figure 6, D and E), but no HCC tumors were identified. Serial biopsies were performed from one pig in group 2 at $6,9,12$, and 22 months. The degree of fibrosis correlated positively $(r=0.85)$ with liver stiffness measured by MRE at the same time points (Figure 7).

\section{Discussion}

$F A H^{-l-}$ pigs are the first genetically modified large-animal model of metabolic liver disease. ${ }^{13,14}$ The acute phenotype of HT1 in $F A H^{-1-}$ pigs, seen after withdrawal of NTBC, closely resembles acute-onset HT1 in humans, which is characterized by acute liver failure and death. ${ }^{14,22}$ In humans, treatment with NTBC abolishes the acute complications of HT1 in most patients. ${ }^{23}$ In this study, we characterized the influence of dosage of NTBC on the chronic phenotype of HT1 in $F A H^{-1-}$ pigs. Based on previous observations, $1 \mathrm{mg} / \mathrm{kg}$ per day NTBC was administered to all pigs during the first 30 days to prevent perinatal morbidity and mortality. ${ }^{14}$ Subsequently, pigs were assigned to groups based on dosing of NTBC. In group 1, pigs were treated with 0.2 to $1 \mathrm{mg} / \mathrm{kg}$ per day NTBC; in group 2, pigs received $0.05 \mathrm{mg} / \mathrm{kg}$ per day, with on/off cycles; and in group 3, pigs received $0 \mathrm{mg} / \mathrm{kg}$ per day. As predicted, lower doses of NTBC led to more severe phenotypic expression.

The most common presentation of HT1 in human infants is the cessation of weight gain and failure to thrive. ${ }^{24,25}$ All $F A H^{-1-}$ pigs demonstrated decreased weight gain compared to WT animals; the most severe phenotypic expression was observed in group 3 animals. Furthermore, the $F A H^{-1-}$ phenotype in the pig model was confirmed with elevation of tyrosine and SUAC in the blood and urine, as seen in the clinical presentation of the disease. More substantial elevations in SUAC were seen in the group 3 animals, consistent with a more severe phenotypic expression. In contrast, tyrosine levels were higher in the group 1 animals, which can be attributed to the mechanism of action of NTBC. ${ }^{26}$

All group 2 and 3 animals developed varying presentations of chronic liver disease, with elevation in their liver biomarkers, ammonia, and international normalized ratio as a marker of synthetic liver function. Postmortem histology demonstrated evidence of bridging fibrosis in the group 2 and long-term group 3 survivors. Animals in group 3, which succumbed early, demonstrated evidence of acute liver failure, as previously reported. ${ }^{14}$ Although HCC is observed in humans and mice, presumably because of mutagenicity of FAA, none of the pigs in this study demonstrated de novo progression to HCC. This is could be, in part, because of the shorter duration of the study (2 years). Regenerative liver nodules were seen in the longest surviving pigs. Future studies with longer follow-up are needed to determine the true incidence of $\mathrm{HCC}$ in $F A H^{-1-}$ pigs. Animals in group 2 undergoing MRE analysis demonstrated clinically significant increases in stiffness. This correlated to the degree of fibrosis and premortem hepatic vein pressure gradient. Recent studies demonstrated the accuracy of MRE in staging fibrosis in humans. ${ }^{27-29}$ This large animal model further validates the use of MRE as a predictor of clinical cirrhosis and worsening portal hypertension. ${ }^{30-32}$

In summary, the $F A H^{-1-}$ pig model represents an exciting and reproducible model for study of chronic liver disease. Withdrawal of NTBC at various time points and dosing strategies led to spontaneous progression of clinically significant liver disease, as evidenced by biochemical markers, ultrasound imaging, MRE, and postmortem histology. Potential applications of a de novo large-animal model of liver cirrhosis include drug development and toxicity testing and evaluating modern imaging technology. The $F A H^{-1-}$ pig model also offers an exciting potential for continuing to test the efficacy of gene therapy. ${ }^{33}$ Future studies include efforts to correct the pig's mutant $F A H$ gene as a preclinical treatment strategy for human patients with HT1 and other inherited metabolic liver diseases. In conclusion, our data demonstrate that $F A H^{-1-}$ pigs will provide a substantial clinical benefit for preclinical testing of pharmaceuticals, imaging modalities, and gene therapy.

\section{Acknowledgments}

We thank Jenny Pattengill (Mayo Clinic Arizona) for histology support; Kristi Simmons (Research and Academic Support Services, Mayo Clinic) for help with manuscript preparation; and Duane Meixner (Mayo Clinic Rochester) for ultrasound support.

F.E. assisted in conducting experiments, collecting data, and writing the manuscript; S.A.M. assisted in conducting experiments, collecting data, and writing the manuscript; J.M.G. assisted in conducting experiments, collecting data, and writing the manuscript; M.Y. performed and analyzed MRE images and assisted in writing the manuscript; I.D.I. analyzed blood samples (amino acid analysis); A.S. assisted in data analysis: P.R. supervised amino acid analysis; B.A. assisted in conducting experiments and collecting data; R.J.M. performed the histological analysis of liver pathology; R.L.E. supervised MRE imaging; M.G. assisted in writing the manuscript; J.B.L. assisted in writing the manuscript; R.D.H. assisted in conducting experiments, collecting data, and writing the manuscript; S.L.N. performed study design, supervised experiments, collected data, and assisted in writing the manuscript.

\section{Supplemental Data}

Supplemental material for this article can be found at http://dx.doi.org/10.1016/j.ajpath.2016.09.013. 


\section{References}

1. Lindblad B, Lindstedt S, Steen G: On the enzymic defects in hereditary tyrosinemia. Proc Natl Acad Sci U S A 1977, 74:4641-4645

2. Liang HC, Shertzer HG, Nebert DW: "Oxidative stress" response in liver of an untreated newborn mouse having a 1.2-centimorgan deletion on chromosome 7. Biochem Biophys Res Commun 1992, 182: $1160-1165$

3. Jorquera R, Tanguay RM: The mutagenicity of the tyrosine metabolite, fumarylacetoacetate, is enhanced by glutathione depletion. Biochem Biophys Res Commun 1997, 232:42-48

4. Manning K, Al-Dhalimy M, Finegold M, Grompe M: In vivo suppressor mutations correct a murine model of hereditary tyrosinemia type I. Proc Natl Acad Sci U S A 1999, 96:11928-11933

5. Lindstedt S, Holme E, Lock EA, Hjalmarson O, Strandvik B: Treatment of hereditary tyrosinaemia type I by inhibition of 4hydroxyphenylpyruvate dioxygenase. Lancet 1992, 340:813-817

6. Larochelle J, Alvarez F, Bussieres JF, Chevalier I, Dallaire L, Dubois J, Faucher F, Fenyves D, Goodyer P, Grenier A, Holme E, Laframboise R, Lambert M, Lindstedt S, Maranda B, Melancon S, Merouani A, Mitchell J, Parizeault G, Pelletier L, Phan V, Rinaldo P, Scott CR, Scriver C, Mitchell GA: Effect of nitisinone (NTBC) treatment on the clinical course of hepatorenal tyrosinemia in Quebec. Mol Genet Metab 2012, 107:49-54

7. Bartlett DC, Lloyd C, McKiernan PJ, Newsome PN: Early nitisinone treatment reduces the need for liver transplantation in children with tyrosinaemia type 1 and improves post-transplant renal function. J Inherit Metab Dis 2014, 37:745-752

8. McKiernan PJ: Nitisinone in the treatment of hereditary tyrosinaemia type 1 . Drugs 2006, 66:743-750

9. Gluecksohn-Waelsch S: Genetic control of morphogenetic and biochemical differentiation: lethal albino deletions in the mouse. Cell 1979, 16:225-237

10. Grompe M, al-Dhalimy M, Finegold M, Ou CN, Burlingame T, Kennaway NG, Soriano P: Loss of fumarylacetoacetate hydrolase is responsible for the neonatal hepatic dysfunction phenotype of lethal albino mice. Genes Dev 1993, 7:2298-2307

11. Al-Dhalimy M, Overturf K, Finegold M, Grompe M: Long-term therapy with NTBC and tyrosine-restricted diet in a murine model of hereditary tyrosinemia type I. Mol Genet Metab 2002, 75:38-45

12. Angileri F, Roy V, Morrow G, Scoazec JY, Gadot N, Orejuela D, Tanguay RM: Molecular changes associated with chronic liver damage and neoplastic lesions in a murine model of hereditary tyrosinemia type 1. Biochim Biophys Acta 2015, 1852:2603-2617

13. Hickey RD, Lillegard JB, Fisher JE, McKenzie TJ, Hofherr SE, Finegold MJ, Nyberg SL, Grompe M: Efficient production of Fah-null heterozygote pigs by chimeric adeno-associated virus-mediated gene knockout and somatic cell nuclear transfer. Hepatology 2011, 54: $1351-1359$

14. Hickey RD, Mao SA, Glorioso J, Lillegard JB, Fisher JE, Amiot B, Rinaldo P, Harding CO, Marler R, Finegold MJ, Grompe M, Nyberg SL: Fumarylacetoacetate hydrolase deficient pigs are a novel large animal model of metabolic liver disease. Stem Cell Res 2014, 13 : $144-153$

15. Aigner B, Renner S, Kessler B, Klymiuk N, Kurome M, Wunsch A, Wolf E: Transgenic pigs as models for translational biomedical research. J Mol Med (Berl) 2010, 88:653-664

16. Bassols A, Costa C, Eckersall PD, Osada J, Sabria J, Tibau J: The pig as an animal model for human pathologies: a proteomics perspective. Proteomics Clin Appl 2014, 8:715-731

17. Cooper DK, Gollackner B, Sachs DH: Will the pig solve the transplantation backlog? Annu Rev Med 2002, 53:133-147

18. Turgeon C, Magera MJ, Allard P, Tortorelli S, Gavrilov D, Oglesbee D, Raymond K, Rinaldo P, Matern D: Combined newborn screening for succinylacetone, amino acids, and acylcarnitines in dried blood spots. Clin Chem 2008, 54:657-664
19. Manduca A, Oliphant TE, Dresner MA, Mahowald JL, Kruse SA, Amromin E, Felmlee JP, Greenleaf JF, Ehman RL: Magnetic resonance elastography: non-invasive mapping of tissue elasticity. Med Image Anal 2001, 5:237-254

20. Wang X, Montini E, Al-Dhalimy M, Lagasse E, Finegold M, Grompe M: Kinetics of liver repopulation after bone marrow transplantation. Am J Pathol 2002, 161:565-574

21. Huang SY, Abdelsalam ME, Harmoush S, Ensor JE, Chetta JA, Hwang KP, Stafford RJ, Madoff DC, Avritscher R: Evaluation of liver fibrosis and hepatic venous pressure gradient with MR elastography in a novel swine model of cirrhosis. J Magn Reson Imaging 2014, 39: 590-597

22. Sniderman King L, Trahms C, Scott CR: Tyrosinemia type I. Edited by Pagon RA, Adam MP, Ardinger HH, Wallace SE, Amemiya A, Bean LJH, Bird TD, Fong CT, Mefford HC, Smith RJH, Stephens K. In GeneReviews [Internet]. Copyright University of Washington, Seattle. 1993. Available at https://www.ncbi.nlm.nih.gov/books/NBK1515. (last revised July 17, 2014).

23. Mayorandan S, Meyer U, Gokcay G, Segarra NG, de Baulny HO, van Spronsen F, Zeman J, de Laet C, Spiekerkoetter U, Thimm E, Maiorana A, Dionisi-Vici C, Moeslinger D, Brunner-Krainz M, LotzHavla AS, Cocho de Juan JA, Couce Pico ML, Santer R, SchollBurgi S, Mandel H, Bliksrud YT, Freisinger P, Aldamiz-Echevarria LJ, Hochuli M, Gautschi M, Endig J, Jordan J, McKiernan P, Ernst S, Morlot S, Vogel A, Sander J, Das AM: Cross-sectional study of 168 patients with hepatorenal tyrosinaemia and implications for clinical practice. Orphanet J Rare Dis 2014, 9:107

24. Russo PA, Mitchell GA, Tanguay RM: Tyrosinemia: a review. Pediatr Dev Pathol 2001, 4:212-221

25. Holme E, Lindstedt S: Diagnosis and management of tyrosinemia type I. Curr Opin Pediatr 1995, 7:726-732

26. Ellis MK, Whitfield AC, Gowans LA, Auton TR, Provan WM, Lock EA, Smith LL: Inhibition of 4-hydroxyphenylpyruvate dioxygenase by 2-(2-nitro-4-trifluoromethylbenzoyl)-cyclohexane-1,3-dione and 2-(2-chloro-4-methanesulfonylbenzoyl)-cyclohexane-1,3-dione. Toxicol Appl Pharmacol 1995, 133:12-19

27. Yin M, Talwalkar JA, Glaser KJ, Manduca A, Grimm RC, Rossman PJ, Fidler JL, Ehman RL: Assessment of hepatic fibrosis with magnetic resonance elastography. Clin Gastroenterol Hepatol 2007, 5: $1207-1213 \mathrm{e} 2$

28. Yin M, Woollard J, Wang X, Torres VE, Harris PC, Ward CJ, Glaser KJ, Manduca A, Ehman RL: Quantitative assessment of hepatic fibrosis in an animal model with magnetic resonance elastography. Magn Reson Med 2007, 58:346-353

29. Huwart L, Sempoux C, Salameh N, Jamart J, Annet L, Sinkus R, Peeters F, ter Beek LC, Horsmans Y, Van Beers BE: Liver fibrosis: noninvasive assessment with MR elastography versus aspartate aminotransferase-to-platelet ratio index. Radiology 2007, 245:458-466

30. Nedredal GI, Yin M, McKenzie T, Lillegard J, Luebke-Wheeler J, Talwalkar J, Ehman R, Nyberg SL: Portal hypertension correlates with splenic stiffness as measured with MR elastography. J Magn Reson Imaging 2011, 34:79-87

31. Yin M, Kolipaka A, Woodrum DA, Glaser KJ, Romano AJ, Manduca A, Talwalkar JA, Araoz PA, McGee KP, Anavekar NS, Ehman RL: Hepatic and splenic stiffness augmentation assessed with MR elastography in an in vivo porcine portal hypertension model. J Magn Reson Imaging 2013, 38:809-815

32. Zhou L, Chen TW, Zhang XM, Yang Z, Tang HJ, Deng D, Zeng NL, Wang LY, Chen XL, Li H, Li CP, Li L, Xie XY, Hu J: Liver dynamic contrast-enhanced MRI for staging liver fibrosis in a piglet model. J Magn Reson Imaging 2014, 39:872-878

33. Hickey RD, Mao SA, Glorioso J, Elgilani F, Amiot B, Chen H, Rinaldo P, Marler R, Jiang H, DeGrado TR, Suksanpaisan L, O'Connor MK, Freeman BL, Ibrahim SH, Peng KW, Harding CO, Ho CS, Grompe M, Ikeda Y, Lillegard JB, Russell SJ, Nyberg SL: Curative ex vivo liver-directed gene therapy in a pig model of hereditary tyrosinemia type 1 . Sci Trans1 Med 2016, 8:349ra99 